\title{
The Effect of the Cusp on the Rate of Convergence of the Rayleigh-Ritz Method
}

\author{
Ioana Sîrbu \\ Harry F. King \\ Department of Mathematics Department of Chemistry \\ 244 Mathematics Building 330 Natural Science Complex \\ SUNY at Buffalo \\ Buffalo, NY 14260-2900 \\ Buffalo, NY 14260
}

\begin{abstract}
This paper investigates how smoothing the Hamiltonian and the cusp of the corresponding eigenfunction affects the rate of convergence of the Rayleigh-Ritz method. A simple example from quantum mechanics is used, with a basis of harmonic oscillator functions.
\end{abstract}

\section{Introduction}

This study is motivated by a computational problem in the electronic molecular structure theory. It has been shown ( 1]) that the variational energy error of a configuration interaction(CI), or any other orbital-based method is slow (of order $O\left(L^{-3}\right)$ or greater, where $\mathrm{L}$ is the maximum angular momentum in the finite orbital basis). This behavior can be explained by the inability of the basis functions to describe the "electron correlation cusps" of the wavefunction introduced by the singularities of the Coulombic potential.

One possible approach that we are exploring is a perturbational one, in which the reference problem has a Hamiltonian free of such singularities, and for which the wavefunctions differ significantly from those of the true Hamiltonian only in the vicinity of such cusps. Traditional CI methods are used to solve the reference problem, and geminal-based methods are employed to solve the low-order Rayleigh-Schrödinger perturbation equations. The success of this approach is dependent upon finding a reference Hamiltonian for which the Rayleigh-Ritz (RR) method converges far more rapidly than for the true Hamiltonian (see [2]).

This paper illustrates how the convergence of the RR method is accelerating with the "smoothing" of the Hamiltonian and of the corresponding groundstate wavefunction for a simple example from quantum mechanics. The singular potential $v$ used here is different from the usual potentials used in quantum chemistry, so we investigate whether the associated operator $H$ is selfadjoint and the RR method for this operator using a basis of harmonic oscillator functions is convergent. 


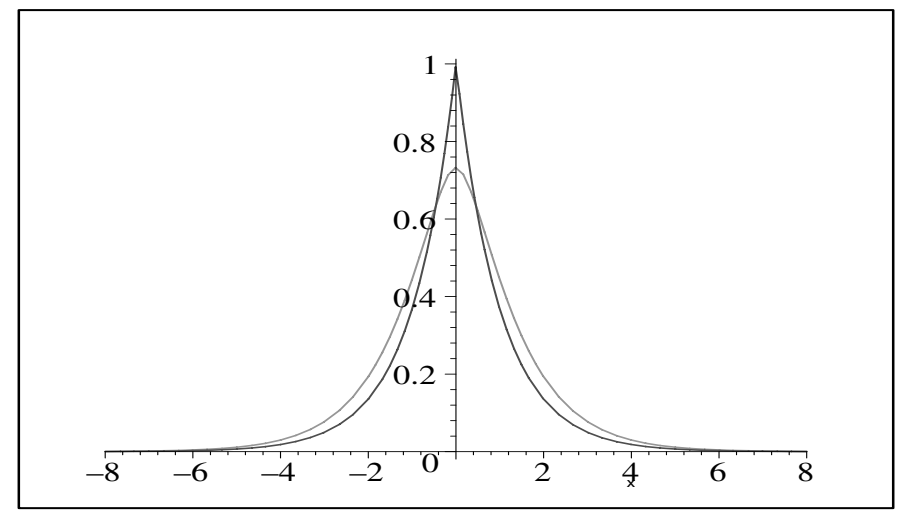

Fig. 1. The wavefunctions $\psi$ and $\psi_{a}$ for $a=1$

\section{The Model}

Consider the one-dimensional Schrödinger equation

$$
-\frac{1}{2} \frac{d^{2} \psi(x)}{d x^{2}}+v(x) \psi(x)=E \psi(x), x \in \mathbf{R}
$$

With the potential $v(x)=-\delta(x)$ the equation ( 1) has the ground-state en$\operatorname{ergy} E=-1 / 2$, with the normalized wavefunction $\psi(x)=\exp (-|x|)$. With the smoothed potential

$$
v_{a}(x)=-\frac{a+1 / 2+a(a+2)|x|+a^{2} x^{2}}{(1+a|x|)^{4}}
$$

the Schrödinger equation has ground-state wavefunction $\psi_{a}(x)=$ $N_{a} \exp \left(-\frac{a x^{2}}{1+a|x|}\right)$ with the same energy $E=-1 / 2 ; N_{a}$ is the normalization constant. The function $\psi(x)$ is continuous, but has a cusp at the origin; the function $\psi_{a}(x)$ has continuous first and second derivatives and a discontinuous third derivative for any $a>0$. Moreover, $\psi_{a}(x) \rightarrow \psi(x)$ pointwise as $a \rightarrow \infty$. $v_{a}(x) \rightarrow 0$ as $a \rightarrow \infty$ for any $x \neq 0$ and $v_{a}(0)=-a-1 / 2 \rightarrow-\infty$ as $a \rightarrow \infty$.

\section{The Expansion}

Consider the expansion of the two wavefunctions, $\psi$ and $\psi_{a}$

$$
\psi(x)=\sum_{n=0}^{\infty} c_{n} \phi_{n}(x), \quad \psi_{a}(x)=\sum_{n=0}^{\infty} c_{n}^{a} \phi_{n}(x)
$$

in the orthonormal basis of harmonic oscillator functions 


$$
\phi_{m}(x)=N_{m} H_{m}(\lambda x) \exp \left(-(\lambda x)^{2} / 2\right),
$$

where $\lambda$ is a positive scaling factor and $N_{m}$ is a normalization constant.

Since both $\psi$ and $\psi_{a}$ are even functions, $c_{2 k+1}=c_{2 k+1}^{a}=0$ for all integers $k$. For even integers we have $c_{2 k}=C_{2 k}$ and $c_{2 k}^{a}=C_{2 k}^{a}$, where

$$
\begin{gathered}
C_{n}=\frac{2 N_{n}}{\lambda} \int_{0}^{\infty} \exp \left(-\frac{x}{\lambda}-\frac{x^{2}}{2}\right) H_{n}(x) d x \\
C_{n}^{a}=\frac{2 N_{n} N_{a}}{\lambda} \int_{0}^{\infty} \exp \left(-\frac{x^{2}}{2}-\frac{a x^{2}}{\lambda^{2}+a \lambda x}\right) H_{n}(x) d x
\end{gathered}
$$

The coefficients $C_{n}$ can be computed using an exact recurrence formula which can be obtained integrating (5) by parts. To compute $C_{n}^{a}$ an approximate quadrature formula is used for the function $f(x)=\exp \left(-\frac{x^{2}}{2}-\frac{a x^{2}}{\lambda^{2}+\lambda a x}\right) H_{n}(x)$, where the roots $x_{j}$ and weights $w_{j}$ are for polynomials orthogonal with respect to the weight function $w(x)=\exp \left(-x^{2}\right)$ on the interval $(0, \infty)$.

Once the expansion coefficients $c_{n}$ and $c_{n}^{a}$ are computed, one can define the projection of the wavefunctions $\psi$ and $\psi_{a}$ on the $n+1$-dimensional space $W_{n}$ spanned by $\phi_{0}, \phi_{1}, \ldots, \phi_{n}$ :

$$
\begin{gathered}
\psi_{n}(x)=\sum_{i=0}^{n} c_{i} \phi_{i}(x), \quad \psi_{n}^{a}(x)=\sum_{i=0}^{n} c_{i}^{a} \phi_{i}(x) . \\
H=-\frac{1}{2} \frac{d^{2}}{d x^{2}}+v(x), \quad H^{a}=-\frac{1}{2} \frac{d^{2}}{d x^{2}}+v_{a}(x) .
\end{gathered}
$$

Next, let us define $E_{n}$ and $E_{n}^{a}$ as the lowest eigenvalue of the Hamiltonian matrix $\left(<\phi_{i}|H| \phi_{j}>\right)_{0 \leq i, j \leq n}$ and of $\left(<\phi_{i}\left|H^{a}\right| \phi_{j}>\right)_{0 \leq i, j \leq n}$, respectively. For each $n$, the norm of the projection $\left\|\psi_{n}\right\|$ is maximized as a function of $\lambda$ and $E_{n}$ is computed for this $\lambda$. Values of $E_{n}$ are reported in Table 1 and $E_{n}^{a}$ are reported in Table 2 for two different values of the smoothing parameter $a$. Also

Table 1. $\lambda$ and the energies $E_{n}^{1}$ and $E_{n}$ for $\psi(x)$

\begin{tabular}{|c|c|c|c|c|}
\hline \multicolumn{5}{|c|}{$\psi$} \\
\hline$n$ & $\lambda$ & $\left(\psi_{n}, \psi_{n}\right)$ & $E_{n}^{1}$ & $E_{n}$ \\
\hline 8 & 1.300 & .997350 & -.405894 & -.409083 \\
16 & 1.450 & .999183 & -.433470 & -.435312 \\
24 & 1.575 & .999629 & -.447763 & -.448960 \\
32 & 1.675 & .999795 & -.456453 & -.457308 \\
40 & 1.750 & .999873 & -.462174 & -.462830 \\
64 & 1.975 & .999955 & -.472857 & -.473204 \\
80 & 2.125 & .999973 & -.477236 & -.477483 \\
104 & 2.275 & .999986 & -.481202 & -.481372 \\
120 & 2.375 & .999990 & -.483174 & -.483311 \\
\hline
\end{tabular}


Table 2. $\lambda$ and the energies $E_{n}^{1, a}$ and $E_{n}^{1}$ for $\psi_{a}(x)$ for $a=4$ and $a=1$

\begin{tabular}{|c|c|c|c|c|c|c|c|c|}
\hline & \multicolumn{5}{|c|}{$\psi_{a}$ for $a=4$} & \multicolumn{4}{c|}{$\psi_{a}$ for $a=1$} \\
\hline$n$ & $\lambda$ & $\left(\psi_{n}^{a}, \psi_{n}^{a}\right)$ & $E_{n}^{1, a}$ & $E_{n}^{a}$ & $\lambda$ & $\left(\psi_{n}^{a}, \psi_{n}^{a}\right)$ & $E_{n}^{1, a}$ & $E_{n}^{a}$ \\
\hline 8 & 1.125 & .999051 & -.485252 & -.485353 & .875 & .999811 & -.498480 & -.498483 \\
16 & 1.225 & .999824 & -.494604 & -.494620 & .950 & .999979 & -.499683 & -.499684 \\
24 & 1.300 & .999947 & -.497446 & -.497450 & 1.00 & .999995 & -.499891 & -.499891 \\
32 & 1.375 & .999979 & -.498464 & -.498467 & 1.05 & .999998 & -.499975 & -.499975 \\
40 & 1.400 & .999989 & -.498934 & -.498938 & 1.075 & .999999 & -.499975 & -.499997 \\
64 & 1.550 & .999998 & -.499752 & -.499752 & & & & \\
80 & 1.625 & .999999 & -.499869 & -.499869 & & & & \\
\hline
\end{tabular}

reported in these tables are energies $E_{n}^{1}$ and $E_{n}^{1, a}$ computed for $\psi_{n}$ and $\psi_{n}^{a}$, the projections of the true wavefunctions on the subspace.

$$
E_{n}^{1}=\frac{<\psi_{n}|H| \psi_{n}>}{\left(\psi_{n}, \psi_{n}\right)}, \quad E_{n}^{1, a}=\frac{<\psi_{n}^{a}\left|H^{a}\right| \psi_{n}^{a}>}{\left(\psi_{n}^{a}, \psi_{n}^{a}\right)}
$$

Note that $E_{n} \leq E_{n}^{1}$ and $E_{n}^{a} \leq E_{n}^{1, a}$. Figures 2 and 3 illustrate the beneficial effects of smoothing.

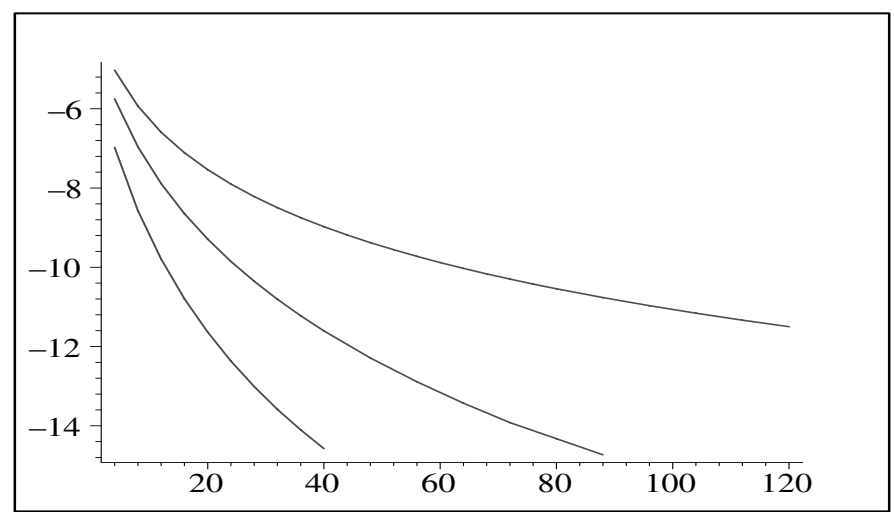

Fig. 2. The norm errors $\ln \left(1-\left(\psi_{n}, \psi_{n}\right)\right)$ (top curve) and $\ln \left(1-\left(\psi_{n}^{a}, \psi_{n}^{a}\right)\right)$ for $a=1$ (lowest curve) and $a=4$ (middle curve) 


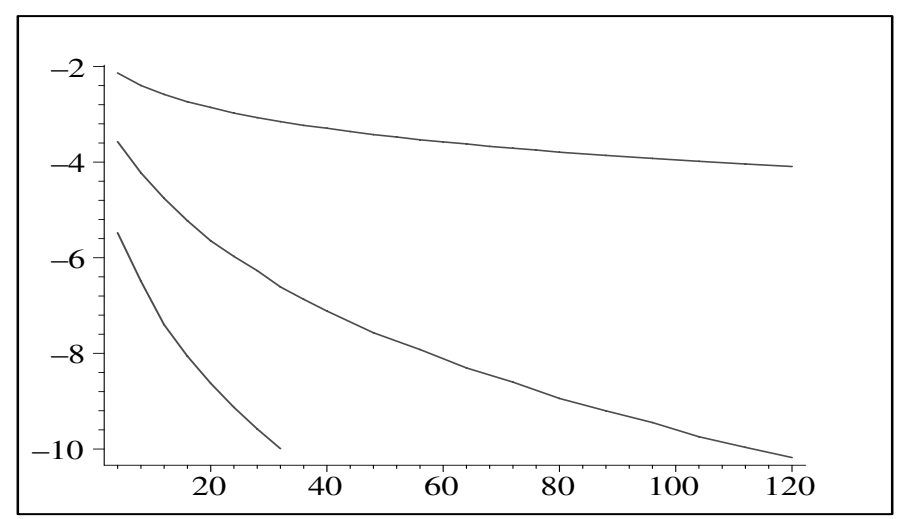

Fig. 3. The energy errors $\ln \left(1 / 2+E_{n}\right)$ (top curve) and $\ln \left(1 / 2+E_{n}^{a}\right)$ for $a=1$ (lowest curve) and and $a=4$ (middle curve).

\section{The Convergence of the RR Method}

For eq. (1) with $v(x)=-\delta(x)$ the behavior of the energy error exhibited in Figure 3 raises a serious question whether the method is just slowly convergent or not convergent at all. In fact, convergence can be established by rigorous mathematics outlined below.

A theorem in Michlin [6] (see also [5]) says that for a positive definite and selfadjoint operator $B$ with the domain $D_{B} \subset L^{2}$ dense in $L^{2}$ the RR method converges to the lowest exact eigenvalue $E_{0}$ of the operator $B$ provided that the basis used $\left\{\phi_{m}\right\}_{m=0,1,2, \ldots}$ is complete in the energy space $H_{B}$. The energy space $H_{B}$ is the closure of $D_{B}$ in the $B$-norm:

$$
\|f\|_{B}=(f, B f)^{1 / 2} .
$$

Let $t$ be the form defined by

$$
t(u, v)=\frac{1}{2} \int u^{\prime}(t) \overline{v^{\prime}(t)} d t+\left(\frac{1}{2}+\delta_{0}\right) \int u(t) \overline{v(t)} d t-u(0) \overline{v(0)}
$$

for functions $u, v \in H^{1}$, where $\delta_{0}>0$. The Sobolev space $H^{1}=\left\{f \in L^{2}, f^{\prime} \in L^{2}\right\}$ (the derivatives are in the generalized sense), is the closure of $C_{0}^{\infty}(\mathbf{R})$ in the $H^{1}$ norm: $\|f\|_{H^{1}}^{2}=\int|f(t)|^{2} d t+\int\left|f^{\prime}(t)\right|^{2} d t$. For any $f \in C_{0}^{\infty}(\mathbf{R})$ and $\epsilon>0$

$$
|f(0)|^{2} \leq \frac{1}{2 \epsilon} \int|f(t)|^{2} d t+\frac{\epsilon}{2} \int\left|f^{\prime}(t)\right|^{2} d t
$$

The inequality ( 12) with $\epsilon=1$ makes it possible to define $f(0)$ for any $f \in H^{1}$ and to prove that the symmetric form $t$ is positive definite $\left(t(u, u) \geq \delta_{0}\|u\|^{2} \forall u \in\right.$ 


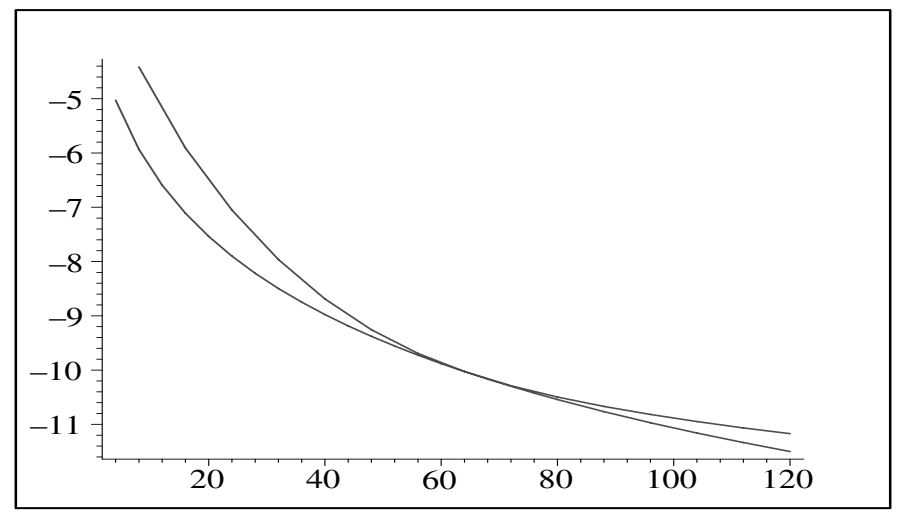

Fig. 4. Norm errors for $\psi(x)$ for $\lambda=2$ (top curve) and optimized $\lambda$ (lower curve)

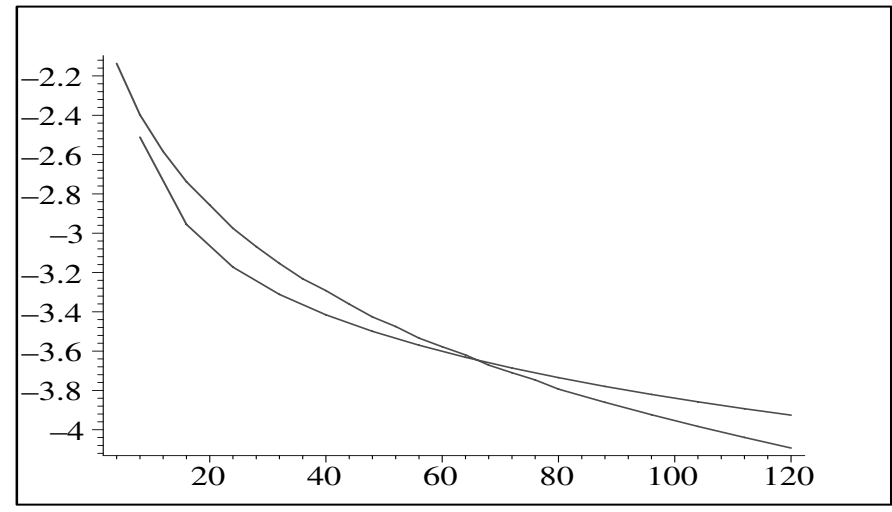

Fig. 5. Energy errors for $\psi(x)$ for xed $\lambda=2$ (upper right) and optimized $\lambda$ (lower right)

$H^{1}, \delta_{0}>0$ ). In its general form, (12) is the main ingredient in proving that the form $t$ is closed. By a representation theorem ( see [3]) there exists a selfadjoint operator $T: D(T) \subset H^{1} \subset L^{2} \rightarrow L^{2}$, positive definite (with the same lower bound $\delta_{0}$ as the form $t$ ) defined by the relation $(T u, v)=t(u, v) \forall u \in D(T), \forall v \in$ $H^{1}$. Moreover, its domain $D(T)$ is dense in the Hilbert space $H^{1}$ with the norm \|\|$_{H_{t}}=t(,)^{1 / 2}$.

This implies that $H_{T}$-the closure of $D(T)$ in the norm \|\|$_{H_{t}}$-is $H^{1}$, since the norms \|\|$_{H_{t}}$ and \|\|$_{H^{1}}$ are equivalent. The basis $\left\{\phi_{m}(x)\right\}_{m=0,1, \ldots}$ is complete in $H^{1}$ for any $\lambda>0$ (see [4) so the RR method is convergent for the operator $T$ and also for $T-1 / 2-\delta_{0}$, which is the operator from eq. (1) with $v(x)=-\delta(x)$. These considerations are for a fixed $\lambda$, while the results from Figure 2 and 3 are for a $\lambda$ varied to optimize $\left(\psi_{n}, \psi_{n}\right)$ for each $n$. The difference between the results for a fixed $\lambda$ and $\lambda$ optimized in the sense above can be seen in Figures 4and [5] As shown in Table [2, $\lambda=2$ maximizes the norm of $\psi_{n}$ for $n \approx 70$, so 
the curves in Figures 4 and 5 coincide at this point. The norm error for optimal $\lambda$ is smaller than that for $\lambda=2$ for all $n$, as expected, while the energy error is lower for $\lambda=2$ for small values of $n$ ( $\lambda$ was optimized with respect to the norm). After $n \approx 70$ the energy error for fixed $\lambda$ is greater than that for optimized $\lambda$. So the method is convergent both for fixed and optimized $\lambda$, but the convergence is very slow due to the inability of the basis functions to describe the cusp $\psi(x)$.

\section{References}

[1] R. N. Hill Rates of Convergence and error estimates formulas for the Rayleigh-Ritz variational method, J. Chem. Phys. 83, 1173-1196 (1985)

[2] H. F. King The electron correlation cusp I.Overview and partial wave analysis of the Kais function, Theoretica Chimica Acta 94, 345-381 (1996)

[3] T. Kato Perturbation Theory for Linear Operators, Springer-Verlag (1980), p.322

[4] T. Kato Fundamental Properties of Hamiltonian Operators of Schrödinger type, Trans. Am. Math. Soc., 195, (1957)

[5] B. Klahn and W. Bingel The Convergence of the Rayleigh-Ritz Method in Quantum Chemistry I. The Criteria of Convergence, Theoretica Chimica Acta 47, 9-26 (1977)

[6] S. G. Michlin Variationsmethoden der Mathematischen Physik, Berlin, Akademie Verlag (1962), p.79 